\title{
Medication Adherence Patterns to Anti-diabetic Drugs among Type 2 Diabetic Patients
}

\author{
Kartick Chanda Shaha *1, Mohammed Asaduzzaman Khan ${ }^{2}$, \\ Farhana Akter ${ }^{3}$, Bhagyoshree Karmokar Jyoti ${ }^{4}$
}

\begin{abstract}
Introduction: The aim of the present study was to evaluate the pattern of medication adherence to anti-diabetic drugs among type 2 diabetic patients at two tertiary level hospitals in Mymensingh. Materials and Methods: An observational, cross sectional study was conducted from January 2016 to June 2016 among patients attending at Endocrine Outpatient Department of the Mymensingh Medical College Hospital and Medicine Outpatient Department of the Community Based Medical College Hospital, after obtaining requisite consent from the patients. Once the consultation by the physician was over, the patients were interviewed. Medication adherence was assessed through the specific four questions patient questionnaire, the modified morisky instrument that has high reliability and validity and the patient was considered to be highly adherent if he or she answered in the negative to all four questions (score-0). Results: In a pool of 300 type 2 diabetics, more than half were female $(n=223,74.3 \%)$. The mean age of the patients were found to be $50.59 \pm 12.57$ years. Less than half $(37 \%)$ of the patients were considered highly adherent, $44 \%$ patients were considered moderately adherent and 19\% patients were considered poorly adherent to the prescribed anti-diabetic drugs. Conclusion: The participants in the area of study were moderately adherent to their anti-diabetic medications. Measures should be taken to improve patient's adherence to the prescribed treatment.
\end{abstract}

Keywords: Adherence, Anti-diabetic drugs.

Number of Tables: 04; Number of Figure: 01; Number of References: 08; Number of Correspondences: 04

*1. Corresponding Author:

Dr. Kartick Chanda Shaha

M.B.B.S, M.phil (Pharmacology)

Lecturer

Department of Pharmacology

Dhaka National Medical College.

Email: Kartick_shaha@yahoo.com

2. Dr. Mohammed Asaduzzaman Khan

Registrar

Department of Medicine

Dhaka National Medical College.

3. Dr. Farhana Akter

Assistant Professor

Department of Anatomy

Dhaka National Medical College.

4. Dr. Bhagyoshree Karmokar Jyoti

Lecturer

Department of Biochemistry

Dhaka National Medical College.

\section{Introduction}

Adherence with medication usage is defined as the proportion of prescribed doses of medication actually taken by a patient over a specified period of time. Compliance a synonymous term which was commonly used in the past implies a passive role and simply following the demands of a prescriber and non compliance has been regarded as associated with deviant or irrational behavior. According to the WHO, non adherence tomedical regimen consist a major clinical problem in the management of patients with chronic illness. Rates of non adherence with any medication treatment may vary from $15 \%$ to $93 \%$, with an average estimated rate of $50 \%{ }^{1}$. Adherence rates to drug regimen in patients with type 2 diabetes are relatively low and vary widely between populations. Poor adherence is an obstacle in therapeutic control of diabetes. There are many factors that could affect patient adherence to drug therapy ${ }^{2}$. Commonly proposed reasons for non adherence to oral medication regimens include forgetfulness and spontaneous activities due to lack of self discipline, limited intelligence or fearless attitude towards the consequences of diabetes ${ }^{3}$. Expectedly, patient non adherence to prescribed hypoglycemic medications could decrease treatment effectiveness with subsequent manifestation of microvascular and macrovascular complications of diabetes ${ }^{4}$. However, to, date, there is no reliable evidence on the pattern of medication adherence to anti-diabetic dugs among type 2 diabetic patients in Bangladesh. The aim of the present study was to evaluate the pattern of medication adherence to anti-diabetic drugs among type 2 diabetic patients at two tertiary level hospitals in Mymensingh. This study undoubtedly benefits the physicians for successful management of diabetes mellitus in the future.

\section{Materials and Methods}

An observational, cross sectional study was conducted from January 2016 to June 2016 among patients 
attending at Endocrine Outpatient Department of the Mymensingh Medical College Hospital and Medicine Outpatient Department of the Community Based Medical College Hospital after obtaining requisite consent from the patients. Total 300 patients were studied during the study period. The interviews were held directly in the corridor just outside the medical Outpatient Department. Prescriptions slips were taken from the patients after taking the written consent and the relevant information was entered into the predesigned proforma to know the pattern of medication adherence to anti-diabetic drugs among type 2 diabetic patients. Medication adherence was assessed through the specific four questions patient questionnaire, the modified morisky instrument that has high reliability and validity and the patient was considered to be high adherent if he or she answered in the negative to all four questions. The patient was considered to be medium adherent if he or she answered in the positive to one or two questions. The patient was considered to be low adherent if he or she answered in the positive to three or four questions. Those four questions were 1) Do you ever forget to take your medicine? 2) Are you careless at times about taking your medicine? 3) If you feel better, do you sometimes stop taking your medicine? 4) Sometimes if you feel worse while taking your medication, do you stop taking them? The study was approved by the institutional ethical committee. Non-Random purposive sampling was employed for collecting data. All filled questionnaires on the pattern of medication adherence to anti-diabetic drugs were entered into the computer for subsequent analysis using SPSS method version 20.1.

\section{Results}

This present study was conducted on 300 type 2 diabetic patients to observe the pattern of medication adherence to anti-diabetic drugs among type 2 diabetic patients at outpatient department of two tertiary level hospitals in Mymensingh. The age structures of the patients have been categorized in years into three groups. Overall 77 (25.7\%) patients were in $\leq 40$ years old while $172(57.3 \%)$ patients were 41-60 years old, $51(17.0 \%)$ patients belong to $>60$ years age group. Most of the patients belonged to the middle age group 41-60 years (Table I). Total numbers of patients both male and female were 300 . It comprised of $77(25.7 \%)$ male and $223(74.3 \%)$ female in outpatient. Female patients were more than the male patients at the outpatient department (Figure 1). Among 300 cases, when the patients were asked about whether they ever forget to take their medicines, 189 patients (63.3\%) answered "yes" and 111 (37\%) answered "no". When asked about whether the patients were not careful in taking their medicines 102 patients (34.0\%) answered "yes" and 198 patients $(66.0 \%)$ answered "no". When they felt better whether they stop taking their medicines, 57 patients (19.0\%) answered "yes" and $243(81.0 \%)$ answered "no" and when they felt worse while taking medication whether they stop taking them, 51 patients $(17.0 \%)$ answered "yes" and $249(83.0 \%)$ answered "no"(Table II). Out of 300 patients, $111(37 \%)$ patients answered no to every question and were considered high adherent (score 0). 131 (44\%) patients answered yes to one or two questions and were considered medium adherent (score 1-2). Only 58 $(19 \%)$ patients answered yes to three or four questions and were considered low adherent (score 3-4) (Table III). Forgetfulness (44.4\%), Carelessness (23.80\%), Financial problem (21.16\%), Meal irregular (3. 17\%), and hypoglycemia $(7.40 \%)$ were considered as major reasons for non adherence to medication. Forgetfulness was the central reason for not taking their medications (Table IV).

Table-I: Age distribution of the study population $(n=300)$.

\begin{tabular}{ll}
\hline Age group $($ years $)$ & Frequency $(\%)$ \\
\hline$\leq 40$ years & $77(25.7)$ \\
$41-60$ years & $172(57.3)$ \\
$>60$ years & $51(17.0)$ \\
Total & $300(100)$ \\
Mean \pm SD & $50.59( \pm 12.57)$ \\
\hline
\end{tabular}

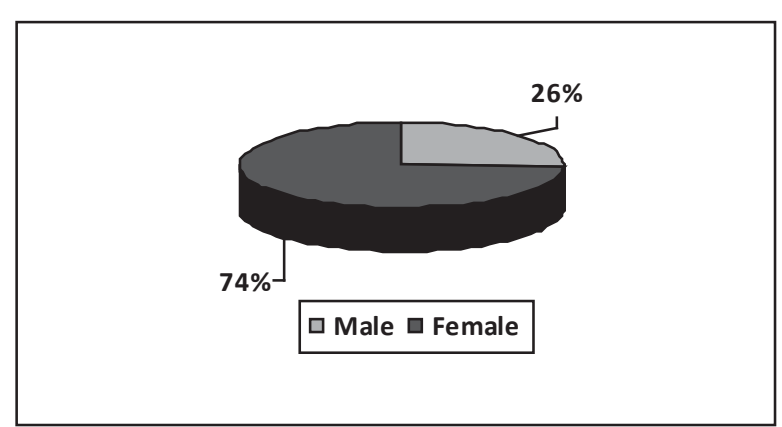

Figure 1: Pie chart showing sex distribution of the study population.

Table-II: Summary of the patient's response to the modified morisky adherence Predictor scale.

\begin{tabular}{lcc}
\hline Patients response & $\begin{array}{l}\text { Frequency } \\
\text { of yes (\%) }\end{array}$ & $\begin{array}{l}\text { Frequency } \\
\text { of no (\%) }\end{array}$ \\
\hline $\begin{array}{l}\text { Do you ever forget to } \\
\text { take your medicine? }\end{array}$ & $189(63)$ & $11(37)$ \\
$\begin{array}{l}\text { Do you sometime not } \\
\text { being careful in } \\
\text { taking your medicine? }\end{array}$ & $102(34)$ & $198(60)$ \\
$\begin{array}{l}\text { If you feel better, do } \\
\text { you sometimes stop } \\
\text { taking your medicine? }\end{array}$ & $57(19)$ & $243(81)$ \\
$\begin{array}{l}\text { Sometimes if you feel } \\
\text { worse when you take } \\
\text { your medication, do } \\
\text { you stop taking them? }\end{array}$ & & \\
\hline
\end{tabular}


Table-III: Distribution of patients according to pattern of medication adherence to anti-diabetic drugs $(n=300)$.

\begin{tabular}{ll}
\hline Adherence pattern (score) & Frequency (\%) \\
\hline High adherence (0) & $111(37)$ \\
Medium adherence (1 -2) & $131(43.66)$ \\
Low adherence (3-4) & $58(19.3)$ \\
\hline Total & $300(100)$ \\
\hline
\end{tabular}

Table-IV: Reasons for non-adherence to anti-diabetic drugs in non compliant patients $(n=189)$.

\begin{tabular}{ll}
\hline Reasons & Frequency (\%) \\
\hline Carelessness & $45(23.80)$ \\
Forgetfulness & $84(44.44)$ \\
Financial problem & $40(21.16)$ \\
Meal irregular & $6(3.17)$ \\
Hypoglycemia & $14(7.40)$ \\
\hline
\end{tabular}

\section{Discussion}

Type 2 diabetes is a chronic disease requiring lifelong treatment, although lifestyle modifications play an important role in diabetes management. This study analyzed the pattern of medication adherence to anti-diabetic drugs among type 2 diabetic outpatients. All together a total of 300 prescriptions were collected during the study period. This study showed that diabetes mellitus is more prevalent in female patients than in male patients. This may be assigned to the fact that women are more obese than men. The other reasons might be due to lack of physical activity, life style changes, dietary habit and stress. Similar results were obtained in the study conducted by Alam et al. (2014), Mann et al. (2009) and Abebaw et al. $(2016)^{5,6,7}$. However other studies reported higher prevalence of DM in men. This study also found a higher prevalence of diabetes was among middle aged patients, with a high percentage $(57.37 \%)$ in the age group of 41-60 years. Mean age $50.59 \pm 12.5$ years. This result correlates with the study of sajith et al. (2014) ${ }^{1}$. A study from the Nepal reported higher mean age 58.1 \pm 11.6 years in type 2 diabetic patients. So this present study does not correlate with the Nepal study. In general elderly patients are at a greater risk of developing type 2 diabetes mellitus. In our study the 4 item modified morisky adherence predictor scale is used to assess medication adherence to anti-diabetic drugs. The assessment of the patient's response to the four item modified morisky adherence predictor scale showed that $37 \%$ of the patients had good adherence with prescribed medications, whereas $43.66 \%$ had medium adherence and $19.3 \%$ had low adherence. Our study findings are quite similar with the findings of a study carried out in India which revealed the following adherence level: good adherence $40 \%$, medium adherence $37.14 \%$ and poor adherence $21.90 \%$. In our study, only $37 \%$ patients answered no to every question and were considered adherent. The adherence rate in this study is higher than adherence rate of $16.66 \%$ reported in Sharma et al. (2014) study ${ }^{8}$. The discrepancy is possibly because of medication adherence measurement method. $44.44 \%$ patients used to miss anti-diabetic drugs due to forgetfulness followed by $23.80 \%$ patients missing anti-diabetic drugs due to their carelessness. The result is consistent with the finding from the Shrestha et al. (2013) study which had reported that, forgetfulness $(42.9 \%)$ as the central reason for not taking their medications ${ }^{3}$. Moreover, the present study showed that $21.16 \%$ of the patients had not adequate cost of medications in relation to the income. High cost of newer oral hypoglycemic agents hindered optimal adherence to the treatment, so one should ensure that cost effective as well as beneficial oral hypoglycemic agents are prescribed to the patients. Our results are not in agreement with the Heissam, Abuamer, and Dahshan, who reported that about $68.62 \%$ of the patients had not adequate cost of medications in relation to the income ${ }^{2}$.

\section{Conclusion}

Type 2 diabetes mellitus being a chronic disorder requires multiple therapeutic approaches including dietary and lifestyle modifications. It is concluded that the participants in the area of study were moderately adherent to their anti-diabetic medications. Measures should be taken to improve patient's adherence to the prescribed treatment for better management of type 2 diabetes mellitus. Various factors of medication non adherence were identified and evaluated. Therefore, we recommend interventions that will address these factors of non adherence in order to improve adherence the more. Some of such interventions include simplifying drug regimen with decreasing the number of drug taken, encouraging patients to monitor their blood glucose level regularly. So continuous patient education and awareness program are required.

Conflict of Interests: None.

\section{Acknowledgements}

The authors are grateful to the entire staff of the medical outpatient department of the Mymensingh Medical College Hospital and Community Based Medical College Hospital, Mymensingh for their cooperation and support during the study period.

\section{References}

1. Sajith, M., Pankaj, M., Pawar, A., et al. Medication adherence to anti-diabetic therapy in patients with type 2 diabetes mellitus. International jounal of pharmacy and pharmaceutical science. 2014; 6(2): 564-570.

2. Heissam, K., Abuamer, Z., Dahshan, N.EL. Patterns and obstacles to oral anti-diabetic medications adherence among type 2 diabetics in Ismailia, Egypt: a cross section study. Pan African medical journal. 2015.

https://doi.org/10.11604/pamj.2015.20.177.4025 
3. Shrestha, SS., Shakya, R., Karmacharya, BM., et al. Medication adherence to oral hypoglycemic agents among type 2 diabetic patients and their clinical outcomes with special reference to fasting blood glucose and glycosylated haemoglobin levels. Kathmandu university medical journal. 2013; 43(3): 226-232.

4. Adisa, R., Alutundu, M.B., Fakeye, T.O. Factors contributing to non adherence to oral hypoglycemic medication among ambulatory type 2 diabetes patients in southwestern Nigeria. Pharm pract. 2009; 7(3): 163-169.

https://doi.org/10.4321/S1886-36552009000300006

5. Alam, M.S., Aqil, M., Qadry, S.A.S., et al. Utilization pattern o oral hypoglycemic agents for diabetes mellitus type 2 patients attending outpatient department at a university hospital in New Delhi. Pharmacology and pharmacy. 2014; 5: 636-645.

https://doi.org/10.4236/pp.2014.57073
6. Mann, D.M., Ponieman, D., Leventhai. H., et al. Predictors of adherence to diabetes medications: the role of disease and medication beliefs. J Behav Med. 2009; 32: 278-284.

https://doi.org/10.1007/s10865-009-9202-y

PMid: 19184390

7. Abebaw, M., Messele, A., Hailu, M., et al. Adherence and associated factors towards anti-diabetic medication among type 2 diabetic patients on follow up at university of Gondar hospital, northwest Ethiopia. Advances in nursing. 2015; Article ID 8579157, 7 pages.

8. Sharma, M., Nazareth, I., Petersen, I. Trends in incidence, prevalence and prescribing in type 2 diabetes mellitus between 2000 and 2013 in primary care: a retrospective cohort study. BMJ Open. 2015

https://doi.org/10.1136/bmjopen-2015-010210 